\title{
Is Data Ownership Empowerment Welfare-Enhancing?
}

\author{
Shilei Li \\ College of Business \\ City University of Hong Kong \\ shileili2-c@my.cityu.edu.hk
}

\begin{abstract}
Under current business models, consumers have to hand over their personal data to "digital giants" in exchange for high-quality services. Should consumers be empowered to have ownership towards the data they generated through their own actions in the platform firm's site? New technologies emerge to empower consumers to control their own data, and the platform firm may need to compensate for the usage of consumers' private data. How consumers and the firm should react to such data ownership empowerment policy, however, is not clear. We build a theoretical model in which consumers have different sensitivities to the loss of data ownership. We show that the impact of data ownership empowerment depends not only on the firm's revenue structure, but also on consumers' need for customized services. The results of the welfare analysis offer managerial implications for policy making.
\end{abstract}

\section{Introduction and Relevant Literature}

When using online platforms (e.g., Google), consumers generate large amounts of data including location information, key words searched, browsing history, IP and cookies, etc. Users usually have no control over their own data; while platform firms, however, are collecting and utilizing such data for their big data enabled customized services such as personalized recommendations, etc., which brings huge revenues to the platform firms, such as Amazon and Harrah's [1] [2], and none of such revenues is shared with the platform users. On one hand, firms can learn users' individual preferences from the data and provide highquality customized services, which usually improves consumer welfare; on the other, consumers are suffering from the loss of data ownership: improper access to personal information and unauthorized secondary use of personal information can make consumers worse off [3]. Unfortunately, users currently have to hand over their

\author{
Juan Feng \\ School of Economics and Management \& \\ Shenzhen International Graduate School \\ Tsinghua University \\ fengjuan@sem.tsinghua.edu.cn
}

personal data to "digital giants" in exchange for online services [4].

Who should own the data that generated in the process when consumers browse, search and make purchase in the firm's site? It is obvious that currently such data is the platform firm's property as they build information systems to track and store such data. Recently, however, some new technologies emerge to empower consumers to control their own data. For example, "Solid" (led by Tim Berners-Lee) gives users freedom to choose whether or not to allow applications to access their own data by decoupling user generated content from the application itself: the data is stored in decentralized "pods" and only available to the applications in the user's "permission list" [4]. Another example is "repay.me". By using block chain technologies to ensure the safety of their data, consumers are empowered to track the revenue generated from their personal data [5]. These technologies make it possible for individual consumers to track and monitor the usage of their own private data, so that the firm needs to compensate for the usage of such private data.

What are the implications of such technologies as well as the empowerment of data ownership? How should consumers decide whether to provide their data to firms in exchange for services, as well as compensation? How should firms optimally adjust the service quality or compensation strategies? Is the empowerment welfare-enhancing? Which firms should apply such data ownership empowerment policy?

This study goes beyond simple privacy protection and examines the impacts of data ownership empowerment. Consistent with existing literature, we argue that firms can manipulate its service quality facing different data policies. We model how a profitmaximizing firm should strategically provide data compensation. In our model, consumer utilities are influenced by both consumer characteristics (e.g., sensitivities towards loss of data ownership) and platform characteristics (e.g., service quality). 
By incorporating firm's cost of providing services with consumers' need for customized services, we find that the impact of data ownership empowerment depends not only on the firm's revenue structure, but also on consumers' need for customized services. Specifically:

1. The firm is not necessarily worse off when consumers are empowered. We identify conditions under which a firm makes more profit from the data ownership empowerment.

2. Contrary to the common belief that consumers benefit from obtaining data ownership, we find that the empowerment un-uniformly affects different consumers. The welfare of consumers who are sensitive to loss of data ownership improves because their rights are protected; however, consumers who are not sensitive to loss of data ownership may be worse off because the quality of service they receive may drop after the empowerment policy implementation.

In the literature, online privacy has been discussed extensively. First, consumers are concerned with the use of their personal data. Surveys indicate that privacy concerns prevent some consumers from using online services [6]. Hann et al. (2007) quantify individuals' valuation toward privacy and find that website privacy protection is worth between US\$30.49 and \$44.62 to U.S. consumers [7]. In addition, surveys and experiments reveal that individuals vary in their sensitivities towards privacy [8]. Following this result, our model differentiates consumers by their sensitivity towards loss of data ownership. Second, Hui et al. (2007) find that individuals could be induced to disclose information by monetary incentives [9]. Our model is consistent in that firms can offer compensation for consumers' private data.

Our study is closely related to several prior studies and generates additional results. Byungwan et al. (2017) study the impact of voluntary profiling on consumer surplus and social welfare [10]. In their monopoly model, consumers can participate in profiling to reduce search cost while losing their privacy. A key difference between their study and ours is that they focus more on voluntary data provision, whereas our model considers the case where the firm can offer compensation to data providers in exchange for data ownership.

Rodrigo et al. (2019) studies the value of personal information in online markets [11]. In their study, a firm can use consumers' private information to price discriminate, and consumers can prevent their data from being used by paying a "privacy cost". Different from their study, in our study, consumers who are empowered do not need to pay for data protection, instead, they get compensated by the firm for using their data. Finally, Jay et al. (2019) build a theoretical model to study the optimal data collection policy [12]. Firm's service quality is not modeled in their model, though. Our model is different in that consumers do NOT need to pay for the online services; instead of firm's pricing strategies, we focus our attention on firm's quality decisions.

Our model makes the first attempt to combine both service quality and data compensation in a framework in which consumers are empowered to have data ownership. The results of the welfare analysis offer managerial implications for policy makers.

\section{Theoretical Model}

We build a theoretical model to examine the impact of empowering consumers to have data ownership. Consider an online platform firm, such as a search engine, online marketplace, social media site, etc., providing free-of-charge online services of quality $t \geq$ 0 . Assume that the cost of providing such services is increasing in the service quality. For simplicity, let $m t^{2}$ represent the cost function, where $m>0$.

Consumers are differentiated by their sensitivity towards loss of data ownership $(\theta)$, where $\theta \sim U[0,1]$. When consumers do not have data ownership, they derive values from the free online services, while their data is asked in return for the free services: the firm is free to collect and make use of their private data, which makes consumers suffer from losing control over their data. Then for a consumer with sensitivity $\theta$, the utility of using the firm's services can be represented by

$$
U(\theta, t)=(1-\theta) t
$$

From this utility function, we can see that consumers' sensitivity towards the loss of data ownership weakly discounts the quality of service provided by the firm. To illustrate, if $\theta=0$, the consumer does not value data ownership and the utility equals to the service quality set by the firm; if $\theta=1$, the consumer is extremely sensitive about the private data being used by the firm, and is indifferent between using the firm's services and leaving.

Because consumers usually generate more traffic and bring more revenue to the firm when they are more satisfied with the online services, we assume that the revenue $(R(\theta, t))$ that the firm generates from a consumer $\theta$ is proportional to the consumer's utility of using the services, and the revenue is higher if the firm is able to use consumers' private data. Without loss of generality, let $R(\theta, t)=(a+b) U(\theta, t)$ if the firm can use the private data, and $R(\theta, t)=a U(\theta, t)$ if the firm cannot, where $a>0$ and $b>0$.

We first consider the case in which the firm is free to use the data generated by consumers. By adding up the revenue generated from each consumer, the firm's 
total revenue can be represented by $R(t)=$ $\int_{0}^{1} R(\theta, t) d \theta=\frac{1}{2}(a+b) t$.

The firm's decision problem is to maximize its profit by adjusting the quality level:

$$
\max _{t} \pi=\frac{1}{2}(a+b) t-m t^{2} \text {. }
$$

\section{lemma.}

Solving the optimal quality, we have the following

\section{Lemma 1 (Equilibrium Outcomes)}

When consumers do NOT have data ownership, the firm's equilibrium quality, the corresponding profit as well as consumer surplus are

$\begin{array}{ll}\text { Service quality } & t_{N}^{*}=\frac{(a+b)}{4 m} ; \\ \text { Firm profit } & P_{N}^{*}=\frac{(a+b)^{2}}{16 m} ; \\ \text { Consumer surplus } & S_{N}^{*}=\frac{(a+b)}{8 m} .\end{array}$

Intuitively, consumers are suffering from the loss of data ownership since their data is being used by the firm. Interestingly, however, Lemma 1 reveals that letting firm utilize such data may sometimes bring benefit to consumers. This is because the equilibrium service quality is increasing in $b$, the per-unit revenue derived from using consumers' private data. That is, when $b$ is large, it is better for the firm to improve the service quality to attract more traffic and therefore, acquire more data generated since the revenue from the data is large. Therefore, when $b$ is large, the extra benefit brought to the consumers by the firm from improving the service quality to attract more data may overcome the negative utility from the loss of data ownership.

Now we analyze the case in which consumers are empowered to have data ownership. That is, the platform firm needs to offer compensation for the usage of the data generated by consumers. Consumers now face two choices: allowing the firm to use their data or not allowing. For data providers, the utility of using the services is represented by $(1-\theta) t$, which is identical to the utility function in the previous case. For consumers who refuse to provide data, there's no data ownership loss. However, because the firm fails to learn their preferences from the data and therefore, cannot provide customized services for them, we assume that they receive a lower-quality service. Then the consumers' utility of using the services without providing data can be represented by $\delta t$, where $\delta \in$ $(0,1)$ represents the ratio of the non-customized service quality to the customized service quality. In addition, we assume that consumer type $\theta$, service quality $t$ as well as $\delta$ are public information.

Following the assumption that firm revenue is proportional to the consumer's utility of using the services, we represent the revenue of the firm by:

$$
R(\theta, t)=\left\{\begin{array}{cl}
(a+b)(1-\theta) t & \text { from data providers; } \\
a \delta t & \text { from other consumers }
\end{array}\right.
$$

Because from equation (1), we obtain that when $\theta<1-\frac{a \delta}{a+b}$, the firm makes more revenue if consumer $\theta$ provides his/her data, we assume that the firm can offer compensation to data providers to encourage consumers to provide data. We study the ideal case, in which the firm can differentiate each consumer type $\theta$ and can provide individualized compensation for each consumer. The firm's optimal compensation strategy is shown in Lemma 2.

Lemma 2 (Firm's Optimal Compensation Strategy and the Equilibrium Consumer Utility)

(1) The firm's optimal compensation strategy is to offer compensation to consumers in the interval $[1-\delta, 1-$ $\left.\delta+\frac{b \delta}{a+b+1}\right]$, and the optimal compensation is given by $C(\theta, t)=(\theta+\delta-1) t$;

(2) In equilibrium, consumer utility function is given by

$$
U(\theta, t)=\left\{\begin{array}{cl}
(1-\theta) t, & \text { if } \theta \leq 1-\delta \\
\delta t, & \text { if } 1-\delta<\theta \leq 1-\delta+\frac{b \delta}{a+b+1} \\
\delta t, & \text { if } \theta>1-\delta+\frac{b \delta}{a+b+1} .
\end{array}\right.
$$

Lemma 2 indicates that in equilibrium, consumers can be divided into three groups: 1. Data providers without compensation $(\theta<1-\delta)$ : consumers who are not sensitive to the loss of data ownership are willing to provide their private data and the firm has no incentive to offer them compensation; 2. Consumers who refuse to provide data $\left(\theta>1-\delta+\frac{b \delta}{a+b+1}\right)$ : the firm would also not offer compensation to these consumers because it would be too expensive. 3. Consumers who receive compensation $\left(1-\delta \leq \theta \leq 1-\delta+\frac{b \delta}{a+b+1}\right)$ : The firm offers compensation to the consumers in the medium range. The three groups of consumers are presented in Figure 1.

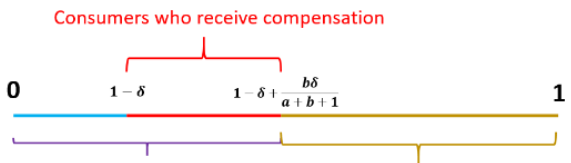

Data providers Consumers who refuse to provide data

Figure 1 Consumers' reactions to data compensation

From the results of Lemma 2, the total compensation offered to consumers can be represented by $\int_{1-\delta}^{1-\delta+\frac{b \delta}{a+b+1}} C(\theta, t) d \theta=\frac{1}{2} t\left(\frac{b \delta}{a+b+1}\right)^{2}$, and by adding up the revenue from each consumer in equation (1), we can obtain that the firm's total revenue is:

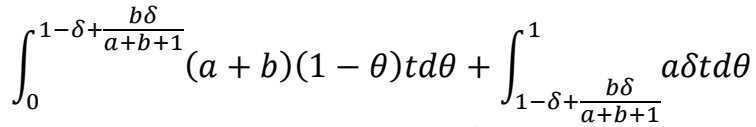

$$
\begin{aligned}
& =\left\{\frac{1}{2}(a+b)\left[1-\left(\delta-\frac{b \delta}{a+b+1}\right)^{2}\right]+a \delta\left(\frac{a \delta+\delta}{a+b+1}\right)\right\} t .
\end{aligned}
$$


Therefore, the firm solves the following problem to maximize its profit:

$$
\begin{gathered}
\max _{t} \pi=\left\{\frac{1}{2}(a+b)\left[1-\left(\delta-\frac{b \delta}{a+b+1}\right)^{2}\right]+\right. \\
\left.a \delta\left(\frac{a \delta+\delta}{a+b+1}\right)-\frac{1}{2}\left(\frac{b \delta}{a+b+1}\right)^{2}\right\} t-m t^{2} .
\end{gathered}
$$

Solving the optimal quality, we have Lemma 3.

\section{Lemma 3 (Equilibrium Outcomes).}

When consumers are empowered to have data ownership, the firm's equilibrium quality, the corresponding profit as well as consumer surplus are:

$$
\begin{array}{lr}
\begin{array}{l}
\text { Service } \\
\text { Quality }
\end{array} & t_{Y}^{*}=\frac{1}{4 m}\left[a+b+\delta^{2}\left(\frac{a^{2}+a-b}{a+b+1}\right)\right] \\
\begin{array}{l}
\text { Firm } \\
\text { Profit }
\end{array} & P_{Y}^{*}=\frac{\left[a+b+\delta^{2}\left(\frac{a^{2}+a-b}{a+b+1}\right)\right]^{2}}{16 m} \\
\begin{array}{l}
\text { Consumer } \\
\text { Surplus }
\end{array} & S_{Y}^{*}=\frac{\left[a+b+\delta^{2}\left(\frac{a^{2}+a-b}{a+b+1}\right)\right]}{8 m /\left(1+\delta^{2}\right)}
\end{array}
$$

\section{The Impact of the Empowerment}

We are interested in examining the impact of data ownership empowerment. By comparing Lemma 1 and Lemma 3, we can obtain the differences in the service quality, firm profit and consumer surplus. For example, we let $\Delta t \stackrel{\text { def }}{=} t_{Y}^{*}-t_{N}^{*}$, then $\Delta t>0$ implies that data ownership empowerment improves the service quality and vice versa.

We first study the impact of the data ownership empowerment on the firm. Intuitively, when consumers are empowered, because some of the consumers require compensation for providing data, the firm is worse off due to the cost of compensation payment for using data. The empowerment, however, can also bring the firm more traffic from the consumers who are sensitive to loss of data ownership since they no longer need to worry about the data ownership loss. Therefore, the impact on the firm depends on the tradeoff between the cost of compensation payment and the extra traffic from the consumers who are sensitive to loss of data ownership.

\section{Proposition 1 (The Impact of the Data Ownership Empowerment on Firm)}

When consumers are empowered to have data ownership, both service quality and firm profit increase if $a+a^{2}>b$, and decrease if $a+a^{2}<b$.

Interestingly, Proposition 1 reveals that the impact of the data ownership empowerment on the firm is contingent on the firm's revenue structure. If the firm revenue from the traffic is relatively large while the revenue from the consumers' private data is relatively small $\left(a+a^{2}>b\right)$, after consumers are empowered to have data ownership, firm revenue from consumers who do NOT value data ownership doesn't reduce too much since $b$ is relatively small, and the benefit of the extra traffic may be more than the loss of the cost of compensation payment. On contrary, the empowerment makes the firm worse off when $b$ is sufficiently large comparing to $a\left(a+a^{2}<b\right)$. That's because in this case, the revenue derived from using consumers' private data is large, while consumers who are sensitive to loss of data ownership refuse to provide the data when they are empowered. In summary, the firm may not be worse off from the data ownership empowerment and the impact on firm depends on the firm's revenue structure.

Next, we examine the impact of data ownership empowerment on consumers. Intuitively, the data ownership empowerment makes consumers better off because they are empowered to have the right to avoid data ownership loss and can also receive compensation. However, Proposition 1 shows that the service quality $t$ changes after the empowerment, which also makes impacts on the welfare of the consumers. If the firm revenue from the traffic is relatively large $\left(a+a^{2}>\right.$ $b$ ), the empowerment of data ownership increases the quality of services, then all consumers benefit from this improvement in quality, which is shown in Figure 2.

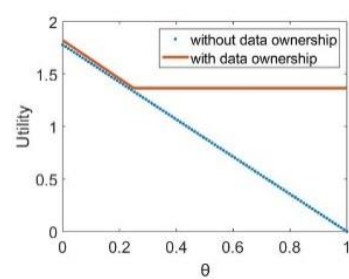

Figure 2 Consumer utility when $a+a^{2}>b$

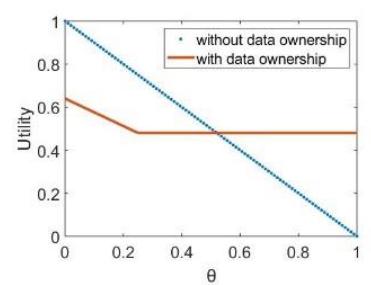

Figure 3 Consumer utility when $a+a^{2}<b$
If the revenue from the consumers' private data is relatively large $\left(b>a+a^{2}\right)$, however, the impact of the data ownership empowerment is more complex. Consumers' rights are protected, as shown in Figure 3, and consumers who are sensitive to the data ownership $\left(\theta>1-\delta+\delta^{3} \frac{b-a-a^{2}}{(a+b+1)(a+b)}\right)$ become better off. However, in this case, empowering consumers leads to a reduction in the service quality $t$, which is harmful to consumers who are NOT sensitive to loss of data ownership $\left(\theta<1-\delta+\delta^{3} \frac{b-a-a^{2}}{(a+b+1)(a+b)}\right)$, and may even reduce the total consumer surplus.

\section{Proposition 2 (The Impact of Data Ownership} Empowerment on Consumers)

The empowerment of data ownership improves consumer surplus in general; However, consumer surplus decreases if $a<\frac{3 \sqrt{2}}{4}-1, \delta>\sqrt{\frac{b^{2}+2 a b+2 a^{2}+2 a}{b-a-a^{2}}}$ and $\frac{1-2 a-\sqrt{1-16 a-8 a^{2}}}{2}<b<\frac{1-2 a+\sqrt{1-16 a-8 a^{2}}}{2}$.

Proposition 2 shows that data ownership empowerment may hurt consumer surplus if the 
empowerment makes the equilibrium quality drop down dramatically. Specifically, because the impact of data ownership on $\Delta t$ (note that $\Delta t=t_{Y}^{*}-t_{N}^{*}=$ $\left.\frac{\delta^{2}}{4 m}\left(\frac{a^{2}+a-b}{a+b+1}\right)\right)$ increases in both $a$ and $\delta$ and decreases in $b$, consumer surplus may decrease when $a$ is small, $b$ is large and $\delta$ is large. Interestingly, Proposition 2 also reveals a sufficiently large $b\left(b \geq \frac{1-2 a+\sqrt{1-16 a-8 a^{2}}}{2}\right)$ ensures that the data ownership empowerment improves overall consumer surplus. This is because in this case, the revenue from consumers' private data is sufficiently large, the firm have to offer a large compensation, which is more than the surplus loss due to the drop in the quality.

\section{Conclusion}

With internet economy grows into a more and more important part of our daily life, data ownership gradually becomes an important issue, that is, who should own the data? Should consumers be empowered and entitled to charge for the firm's usage of their private data? This paper examines the impact of empowering consumers with data ownership on both of the firm and consumers. Our theoretical model studies the optimal strategies of firm and consumers facing different data ownership policies. It turns out that the impact depends not only on the firm's revenue structure, but also on consumers' need for customized services. When consumers are empowered to have data ownership, consumer surplus increases in general; surprisingly, however, they may also be worse off if the value of the private data to the firm is moderate, the firm's revenue from its traffic is low and consumers' need for customized services is low. The firm is better off if the per-unit revenue from the private data is low compared to the per-unit revenue generated from the traffic, and worse off otherwise.

Our work is not without limitation. First, we only study the monopoly case. That is, the firm has sufficient market power to change the quality and compensation policies. A possible future direction is to study a competitive market or a duopoly market. Second, we only examine the case that the firm has linear revenue function with a quadratic cost function. Future work can also study different types of revenue and cost functions. Third, in this paper, consumers only have two choices: to either provide or refuse to provide all of their private data. Future work may relax this restriction, and allow consumers to provide only part of their data. Fourth, our theoretical model reflects a relatively new phenomenon and so far there is few empirical data available. Field experiments can be carried out for future work to test the validity of our work.

\section{Technical Details}

We provide the proof details of the lemmas, propositions and corollaries bellow.

\section{Proof of Lemma 1}

$$
\text { Let } \frac{\partial \pi}{\partial t}=0 \text {, we get } t_{N}^{*}=\frac{(a+b)}{4 m} \text { and } \frac{\partial^{2} \pi}{\partial t^{2}}=-2 m<0
$$
makes sure that it is the firm's optimal quality.

Consumer surplus $S(t)=\int_{0}^{1} U(\theta, t) d \theta=\frac{1}{2} t$. Plugging $t_{N}^{*}=\frac{(a+b)}{4 m}$ into consumer surplus function and profit function, the results are the equilibrium consumer surplus and firm profit respectively.

\section{Proof of Lemma 2}

(1) For $\theta \geq 1-\delta$, if the compensation $C(\theta, t)<$ $(\theta+\delta-1) t$, consumers would refuse to provide data since $(1-\theta) t+C(\theta, t)<\delta t$, which means the utility of providing data is smaller. As a result, the compensation must be at least as large as $(\theta+\delta-1) t$. Also, the compensation cannot be larger than $(\theta+\delta-$ 1) $t$ because it cannot bring more revenue to the firm.

(2) $\theta \leq 1-\delta+\frac{b \delta}{a+b+1}$ if and only if $(a+b)(1-$ $\theta) t-(\theta+\delta-1) t \geq a \delta t$ and therefore, it is worthwhile for the firm to offer compensation to the consumers.

(3) It equals to the summation of the utility of using the services and the compensation.

\section{Proof of Lemma 3}

$$
\text { Let } \frac{\partial \pi}{\partial t}=0 \text {, we get } t_{Y}^{*}=\frac{1}{4 m}\left[a+b+\delta^{2}\left(\frac{a^{2}+a-b}{a+b+1}\right)\right]
$$
and $\frac{\partial^{2} \pi}{\partial t^{2}}=-2 m<0$ makes sure that it is the firm's optimal quality.

Consumer surplus $S(t)=\int_{0}^{1} U(\theta, t) d \theta=\frac{1}{2}(1+$ $\left.\delta^{2}\right) t$. Plugging $t_{Y}^{*}$ into consumer surplus function and profit function, the results are the equilibrium consumer surplus and firm profit respectively.

\section{Proof of Proposition 1}

Let $\frac{t_{Y}^{*}}{t_{N}^{*}}>1$, and it is equivalent to $\delta^{2}\left(\frac{a^{2}+a-b}{a+b+1}\right)>0$. Since all $a, \mathrm{~b}$ and $\delta$ is positive parameters, we know that $\frac{t_{Y}^{*}}{t_{N}^{*}}>1$ if and only if $a+a^{2}>b$.

Similarly, $\frac{t_{Y}^{*}}{t_{N}^{*}}<1$ if and only if $a+a^{2}<b$.

\section{Proof of Proposition 2}

It is easy to know that $\frac{S_{Y}^{*}}{S_{N}^{*}}<1$ is equivalent to $\delta>$ $\sqrt{\frac{b^{2}+2 a b+2 a^{2}+2 a}{b-a-a^{2}}}$. Note that $\delta \leq 1$, so $\frac{b^{2}+2 a b+2 a^{2}+2 a}{b-a-a^{2}}<$ 1 must hold if $S_{Y}^{*}<S_{N}^{*}$. 
Next, we show that when $\frac{S_{Y}^{*}}{S_{N}^{*}}<1, \frac{b^{2}+2 a b+2 a^{2}+2 a}{b-a-a^{2}}<$ 1 if and only if $a<\frac{3 \sqrt{2}}{4}-1$ and $\frac{1-2 a-\sqrt{1-16 a-8 a^{2}}}{2}<$ $b<\frac{1-2 a+\sqrt{1-16 a-8 a^{2}}}{2}$.

We claim that $b-a-a^{2}>0$ when $S_{Y}^{*}<S_{N}^{*}$. That's because if $b-a-a^{2} \leq 0$, the quality of the services improves when consumers are empowered to have data ownership and consumer surplus cannot decline for every $\theta$. This contradicts $S_{Y}^{*}<S_{N}^{*}$.

The positive denominator $\left(b-a-a^{2}>0\right)$ makes sure that $\frac{b^{2}+2 a b+2 a^{2}+2 a}{b-a-a^{2}}<1$ is equivalent to $b^{2}-(1-$ $2 a) b+3 a(a+1)<0$. Note that equation $b^{2}-(1-$ $2 a) b+3 a(a+1)=0$ has two roots $\left(\frac{1-2 a-\sqrt{1-16 a-8 a^{2}}}{2}\right.$ and $\left.\frac{1-2 a+\sqrt{1-16 a-8 a^{2}}}{2}\right)$ when $a<\frac{3 \sqrt{2}}{4}-1$. Therefore, $\frac{b^{2}+2 a b+2 a^{2}+2 a}{b-a-a^{2}}<1$ if and only if $a<\frac{3 \sqrt{2}}{4}-$ 1 and $\frac{1-2 a-\sqrt{1-16 a-8 a^{2}}}{2}<b<\frac{1-2 a+\sqrt{1-16 a-8 a^{2}}}{2}$ given that $S_{Y}^{*}<S_{N}^{*}$.

\section{References}

[1] Davenport TH, "Competing on analytics", Harvard Business Review, 84(1): 98-107.

[2] Brynjolfsson E, McAfee A, "The Second Machine Age: Work Progress, and Prosperity in a Time of Brilliant Technologies", WW Norton \& Company, New York, 2014.

[3] Smith H. J., S. Milberg, S. Burke, "Information privacy: Measuring individuals' concerns about organizational practices”, 1996, MIS Quart, 20(2): 167-196.
[4] Tim Berners-Lee, "One small step for the web", 2018, https://inrupt.com/blog/one-small-step-for-the-web.

[5] Angel Milev, "Blockchain startup can help consumers: profit from their personal data", January 2018, https://cointelegraph.com/news/blockchain-startup-canhelp-consumers-profit-from-their-personal-data.

[6] Privacy \& American Business (P\&AB), "New survey reports an increase in ID theft and decrease in consumer confidence", Conducted by Harris Interactive, 2005, http://www.pandab.org/ deloitteidsurveypr.html.

[7] Hann I. H., K. L. Hui, T. Lee, I. Png, "Overcoming online information privacy concerns: An information processing theory approach", J. Management Inform. Systems, 2007, 24(2) 13-42.

[8] Acquisti A., J. Grossklags, "Losses, gains, and hyperbolic discounting: An experimental approach to information security attitudes and behavior", Proc. 2nd Workshop Econom. Inform. Security (WEIS '03), College Park, MD, 2003.

[9] Hui K.-L., H.-H. Teo, S.-Y. T. Lee, "The value of privacy assurance: An exploratory field experiment”, MIS Quart, 2007, 31(1): 19-33.

[10] Koh Byungwan, Srinivasan Raghunathan, and Barrie R. Nault, "Is voluntary profiling welfare enhancing?" Management Information Systems Quarterly, 2017.

[11] Montes R, Sand-Zantman W, Valletti T, "The value of personal information in online markets with endogenous privacy”, Management Science, Mar 2019, 65(3): 134262.

[12] Choi, Jay Pil, Doh-Shin Jeon, and Byung-Cheol Kim, "Privacy and personal data collection with information externalities", Journal of Public Economics, 2019, 173: 113124 